\title{
The near triad and associated visual problems ${ }^{\dagger}$
}

\author{
R Emslie*, A Claassens*, N Sachs* and I Walters* \\ Department of Optometry, University of Johannesburg, Auckland Park Campus, PO Box 524, \\ Auckland Park, Johannesburg, 2006 South Africa
}

\section{<ingridwalters@webmail.co.za>}

When a person changes fixation from one target, at a given fixation distance, to another target, at an alternate fixation distance, a number of ocular systems need to be altered in order to maintain clear single binocular vision. One such system is that of accommodation, involving either positive or negative accommodation ${ }^{1}$. When viewing an object at six meters, a distance known optometrically as optical infinity, it is assumed that parallel light rays enter the eye. For an emmetrope this results in zero accommodative demand and therefore zero accommodation. When viewing an object closer than 6 meters positive accommodation is required. This involves intraocular lens changes which ultimately increase the refractive power of the eye, allowing the rays to form a point focus on the retina. The opposite process, negative accommodation, occurs when an object is fixated further away from some near fixation point. Accommodation can therefore be thought of as the process by which the refractive power of the eye is altered to ensure a clear retinal image ${ }^{1}$.

It should also be noted that a change in the position of the visual axes must also take place ${ }^{1}$. This occurs to ensure that a single image of the target is seen. The change in relative position of the visual axis is called convergence when the angle formed by the visual axes increases, and divergence when this angle decreases ${ }^{1}$.

When a person fixates a near target an additional process takes place, that is, pupillary constriction. This occurs together with accommodation and convergence, and alters the depth of focus ${ }^{1}$. These three sub-systems work together as a synkinesis known as the near triad ${ }^{1,2}$.

One should not forget the fact that the processes mentioned above are very complex and involve numerous pathways, the details of which are little known. As a result it is easy to understand that many problems can occur in each process, resulting in visual problems. This paper serves to address some of those visual problems.

\section{Accommodation}

The amplitude of accommodation represents the closest near focusing response that can be produced with maximal voluntary effort in the fully corrected eye ${ }^{2}$. From around five years of age to around 52 years of age, the accommodative amplitude progressively decreases at a rate of approximately $0.30 \mathrm{D}$ per year ${ }^{2}$. Various theorists attribute the loss of accommodation to changes in the elastic properties of the lens, changes in the action of the ciliary muscle, or both ${ }^{3}$. The anterior chamber depth, shape of the lens, refractive index and position of the lens surfaces change with age, affecting the accommodative process ${ }^{4}$. This leads to the development of presbyopia. The amplitude of accommodation inevitably decreases from $11 \mathrm{D}$ or more at age 10 to nearly $0 \mathrm{D}$ by age 70 years. This means that an emmetropic child can focus an object as close as $10 \mathrm{~cm}$, while an older adult must move objects at least a metre or more away to see them clearly ${ }^{3}$.

\footnotetext{
${ }^{\dagger}$ This paper was prepared as part of the Binocular Vision B course under the supervision of Professor WDH Gillan.

*BOptom students

Received 7 September 2007; revised version accepted 1 November 2007
} 
Accommodative insufficiency occurs when accommodation is persistently lower than expected for the patient's age $^{2}$. These patients will usually exhibit a reduction in the accommodative amplitude by $2 \mathrm{D}$ or more ${ }^{5}$. They also show a reduced positive relative accommodation, difficulty with negative lens of accommodative facility and an increased lag of accommodation ${ }^{6}$. The main symptom in accommodative insufficiency is general asthenopia related to near work. On occasion a patient might have sufficient amplitude of accommodation, but is unable to maintain the response. This may be the first stage of accommodative insufficiency, and has also been referred to as accommodative fatigue ${ }^{2}$. In such cases patients will report that their work is clear when they initially look at it, but after some time it starts to blur.

Accommodative excess can be defined as accommodation that is persistently higher than expected for the patient's age $^{2}$. Here patients will also exhibit a lead of accommodation and reduced negative relative accommodation $^{6}$. It is often secondary to convergence insufficiency ${ }^{2}$. Accommodative excess can also be regarded as an inability to relax accommodation, known as a spasm of accommodation. The patient will usually report bilateral blurring and fluctuating vision, while headaches, ocular pain, micropsia, macropsia and diplopia may also occur ${ }^{7}$. With accommodative spasm the refractive power of the eye is increased and a hyperopic patient will appear to be less hyperopic, an emmetropic patient will appear to be myopic, and a myopic patient will appear to be more myopic ${ }^{7}$.

Accommodative spasm is usually bilateral, however, Rutstein and Marsh-Tootle ${ }^{7}$ report a case in which a healthy 27 year old female presents with a unilateral accommodative spasm. The accommodative spasm was triggered by occlusion of the contralateral eye causing transient unilateral visual loss. Furthermore, after LASIK, a patient can develop an accommodative $\operatorname{spasm}^{8}$ which changes the spherical aberration to negative ${ }^{9}$. In addition, it should be noted that when a person accommodates, the spherical aberration also becomes negative ${ }^{10}$.

According to Currie and Manny ${ }^{11}$ when young infants attend a near target their accommodative system brings the near object into reasonable focus on the retina, but leaves farther objects out of focus. Further maturation in the programming and execution of the motor response, as well as developing the interpretation of accommodative cues, allow the accommodative response to be refined. The rate of accommodation varies among infants, and one factor that affects the rate is the presence of significant hyperopia.

Accommodative infacility occurs when the accommodative dynamics, that is, latency, time constant, and peak velocity are slowed. Changes in accommodation only occur with effort and difficulty in the presence of normal response magnitude 2 . Patients exhibiting this condition usually complain of near-to-far or far-to-near blur. A number of systemic drugs can result in the above-mentioned conditions occurring. For example, alcohol, phenothiazides, antihistamines and central nervous system stimulants can lead to accommodative insufficiency, while morphine, sulfonamides and carbonic anhydrase inhibitors can lead to an accommodative excess ${ }^{2}$. It is important to note the above and their effects on accommodative functioning. One should make a point of asking patients about medications they may be taking, as the majority might not associate their visual problem to their medications.

It should also be noted that a variety of disease-related peripheral and central neurological conditions, as well as systemic and ocular based conditions can also adversely affect accommodation ${ }^{2}$. For example sinusitis, anemia, herpes zoster, measles and glaucoma, to name but a few, can cause accommodative insufficiency, while encephalitis, syphilis and head trauma can lead to an accommodative excess ${ }^{2}$. According to Al-Qurainy ${ }^{12}$, approximately $20 \%$ of people with traumatic brain injury have an accommodative dysfunction. They also exhibit reduced fusional-vergence ranges, with or without a normal nearpoint of convergence ${ }^{3}$. Accommodation does not develop in many children with vision impairments who experience a blurred image, or in many children with Down Syndrome or Cerebral Palsy, even those whose visual acuity at distance is normal or near normal ${ }^{1}$. With such children it is necessary to prescribe spectacles for near activities ${ }^{3}$. Another example is Adie syndrome. These patients can have an equal amplitude of accommodation monocularly, but binocularly the accommodative response for the affected eye is reduced ${ }^{13}$. Care should be taken to monitor these patients, as the unequal amplitude can lead to the development of amblyopia. 
Lag of accommodation can be thought of as under-accommodation, where the accommodative response is less than the accommodative demand ${ }^{14}$. Therefore, the accommodative system postures at a point distal to the plane or target of regard. This serves as an error signal, allowing the accommodative system to maintain a certain amount of accommodation for a given target distance. Clinical experience has shown that normal, asymptomatic patients tend to show a $+0.50( \pm 0.25)$ DS accommodative lag under binocular conditions ${ }^{2}$. If the lag of accommodation is greater that the above mentioned quantity, a high lag of accommodation results. This can be caused by accommodative dysfunction (insufficiency, fatigue, paresis, infacility), hyperopia or latent hyperopia, esophoria and poor negative vergences or the patient could be over-minused ${ }^{2}$. A lead of accommodation occurs when there is over-accommodation. Here the accommodative system postures at a point proximal to the plane or target of regard and the accommodative response is greater than the accommodative demand. A low lag or lead of accommodation can be caused by accommodative dysfunction (accommodative spasm or spasm of the near reflex), exophoria and poor positive vergences, or the patient could be overplussed $^{2}$. Spherical aberration has an affect on the demand and the defocus of accommodation, leading to either a lag or a lead of accommodation ${ }^{10}$. When an emmetrope accommodates, it results in a lag detected in the horizontal meridian as well as with-the-rule astigmatism. The etiology is unknown ${ }^{15}$.

Accommodation plays a role in the computer vision syndrome. Extensive use of a computer can lead to either reduced accommodation or over-accommodation resulting in eyestrain. It has also been shown that it reduces the amplitude of accommodation ${ }^{16}$. Prolonged accommodation due to near work, as occurs during extensive computer work, increases the prevalence of myopia or pseudo-myopia ${ }^{9,16}$. The quality of the display also affects the accommodative response where a more blurred image will result in a lag of accommodation. Another factor that reduces the accommodative amplitude is the luminance of the workplace ${ }^{16}$, which explains why it is so important to have appropriate lighting in the work place. Staff should also be encouraged to take breaks regularly in an attempt to help relieve the strain on the visual system ${ }^{16}$.

Anisometropia or unequal visual stimulus can cause unequal accommodation. This is thought to exist so that fine stereopsis can be maintained and to help the process of making the refractive errors in both eyes equal. Unequal accommodation is not due to pupil constriction and is in response to a static target. It tends to decrease at the point of the dark focus ${ }^{17}$.

An esotropia caused by an increased accommodative effort or an abnormally high AC/A ratio is referred to as accommodative esotropia ${ }^{1}$. It is the best recognized binocular disorder with clear implications for refractive prescription ${ }^{1}$. Many cases of accommodative esotropia present around three years of age and is associated with hyperopia, astigmatism and anisometropia ${ }^{1,2,18}$. The main characteristics of accommodative esotropia are that the deviation is completely relieved by the correction for hyperopia, where the full cycloplegic refractive correction is prescribed for constant wear ${ }^{19}$. If it is not picked up early enough, the constant deviation can result in the development of amblyopia and anomalous retinal correspondence ${ }^{18}$. In such cases, management involves correction of the refractive error and treatment for the amblyopia.

\section{Convergence}

Convergence, which is mainly a reflex activity as postulated by Maddox $^{20}$, is considered to have four components. These include, proximal convergence, which occurs in response to the perceived nearness of an object, fusional convergence, which occurs to maintain single binocular vision in the presence of disparate retinal images, tonic convergence, which is maintained by the tonus of the extra-ocular muscles and finally accommodative convergence, which is mainly reflex convergence that is brought about when active accommodation occurs. Convergence can also be initiated voluntarily ${ }^{18,20}$ in the absence of a near stimulus. This reaction is controlled by the frontal oculomotor cortex whereas reflex convergence is controlled by the occipital cortex ${ }^{18,21}$.

Convergence and its relationship with accommodation can be expressed linearly as the AC/A ratio, which is the amount of accommodative convergence expressed in prism dioptres which occurs per dioptre of accommodation $^{2,18,20}$. It has been suggested that the AC/A ratio stays roughly the same throughout life, 
suggesting that it is the stimulus for accommodation that drives the accommodative convergence response rather than the amount of accommodation which decreases with age ${ }^{18,22}$. There are a number of different methods used in the calculation of the AC/A ratio, the most commonly used ones being the heterophoria/tropia method, the gradient and the fixation disparity methods ${ }^{2,18}$. The AC/A ratio is significant as it is useful in the diagnosis of various binocular vision syndromes. It has an influence on the type of phoria (or tropia) and plays a role in the choice of treatment ${ }^{18}$.

It has been found that when the accommodative response in myopes is decreased, the AC/A ratio is high and there is an esophoria at near, the chance of myopia progression is high ${ }^{23}$. In order to preserve binocularity, a patient with a near esophoria must relax their accommodation to subsequently decrease the accommodative convergence response. Under-accommodation at near leads to hyperopic retinal defocus. In the process of emmetropization the eyes' axial length increases to compensate for the focus falling behind the retina and induces myopia progression ${ }^{23}$.

\section{Convergence anomalies}

Problems related to convergence can be identified after accommodative functioning has been tested and no uncorrected ametropia exists ${ }^{2}$. Duane ${ }^{24}$ described and classified four types of binocular commitant convergence syndromes. If an esodeviation (phoria or tropia) is larger at near than at distance and the AC/A ratio is higher than normal, a convergence excess is said to exist. If the esodeviation is greater at distance and a low $\mathrm{AC} / \mathrm{A}$ is present, a divergence insufficiency is said to exist. If an exodeviation is greater at near than at distance, in combination with a lower than normal $\mathrm{AC} / \mathrm{A}$ ratio, then a convergence insufficiency exists. And finally, if the exodeviation is greater at distance and the $\mathrm{AC} / \mathrm{A}$ is higher than normal, a divergence excess exists ${ }^{1,2,24}$. It should be noted that divergence excess and insufficiency types are far point syndromes and will not be discussed further.

Additional anomalies of convergence, namely convergence paralysis and convergence spasm can also occur $^{18}$. Convergence paralysis is the inability to converge which is commonly attributed to neurological lesions in the areas of the third nerve nucleus, Corpa Quadrigemina or pineal gland (as in Parinaud's syndrome $)^{18,25}$. Symptoms include crossed diplopia, which presents worse at near, and there is usually a reduction in accommodation $^{18}$. Convergence spasm is a state of over-convergence which is always accompanied by an accommodative spasm. The symptoms are equal to that of an accommodative spasm and can include headaches, general ocular discomfort and blurring of vision but diplopia will usually also be reported ${ }^{2,18}$.

A patient with a convergence excess syndrome would be markedly esophoric at near, have an AC/A ratio greater than normal (more than $6 \mathrm{pd} / \mathrm{D}$ ) and would have reduced negative fusional convergence reserves at near. At the far point the patient would have a phoria close to ortho and adequate reserves ${ }^{1,2,24}$. In severe cases of convergence excess the patient may develop near intermittent esotropia and suppression ${ }^{2}$. Symptoms include uncomfortable near vision, inability to read for extended periods of time, asthenopia, tired/strained eyes, frontal headaches and occasional diplopia ${ }^{2,18}$. Treatment options are controversial and patient specific. Firstly, base-out prism can be used to compensate for the esophoria and satisfy Sheard and Percival's criteria at near. However, the adaptation to prism needed and the distortions created make prism correction unpopular. Most commonly used is the combination of an add at near, either a bifocal or a pair of reading glasses and visual exercises that train and improve the negative fusional ranges at the near point. The orthoptic training is dependent on patient age and motivation. The time factor also needs to be taken into account when deciding on a suitable treatment plan ${ }^{2}$.

A patient with a convergence insufficiency syndrome would have a marked near exophoria, a low AC/A ratio (less than $4 \mathrm{pd} / \mathrm{D}$ ) and reduced positive fusional convergence reserves. The patient will also have a receded near point of convergence, greater than $6 \mathrm{~cm}$ from the nose ${ }^{26,27}$. At distance the patient would present with orthophoria or a small exophoria and adequate convergence reserves ${ }^{1,2,18}$. Often patients over-accommodate in an attempt to drive accommodative-convergence and aid fusion ${ }^{2}$. Symptoms include frontal headaches, blurred near vision, asthenopia, tired eyes, irritation and grittiness when reading and occasional horizontal diplopia. 
Patients will often close one eye when reading and performing near-related tasks or avoid them completely ${ }^{18,26}$. Convergence insufficiency responds very well to vision training and orthoptic exercises. The aims are to improve convergence and increase the convergent fusional ranges. Alternatively base-in prism can be prescribed to decrease the stress on the convergence system and would require minimal time from both patient and clinician $2,18,26$.

\section{Pupillary reactions}

Pupillary reactions are an indirect and objective manner of quantitatively and qualitatively measuring visual function. It makes it possible to identify optic nerve disease as well as its general location in relation to the lateral geniculate body (LGB) ${ }^{21}$. The pupillary reflex consists of an afferent and efferent loop. In the afferent loop, information runs in the same retinal ganglion cell axons as the visual information ${ }^{21}$. However, it was documented by Kardon ${ }^{28}$ that there is a lack of exact affinity between anterior visual pathway vision loss and pupil reactions. This led him to believe that there are two subsystems that run along the anterior visual pathway and use two separate divisions of the ganglion cells therein. The afferent loop fibres then branch off rostraly before the LGB and synapse in the pretectal nuclei. The neural impulses are then conducted to pretectal nuclei on either side of the Edinger-Westphal Nucleus ${ }^{21}$. The efferent loop is a two neuron pathway, running from the Edinger-Westphal Nucleus via the parasympathetic nerve fibres of the third cranial nerve, the oculomotor nerve, which then synapse in the ciliary ganglion. These neural impulses are then conducted via short ciliary nerves to the iris and ciliary body ${ }^{21}$. When positively stimulated, these impulses cause pupil constriction and positive accommodative responses ${ }^{29}$.

\section{Near pupil responses}

The stimulation of the near pupil response for a proximal fixation object has undergone much debate. Marg and Morgan ${ }^{30}$ deduced that the near pupil response is stimulated by accommodation and not by convergence. Phillips et $a l^{31}$ stated that target misalignment and an increase in perceived target size (magnification) at near were the driving forces behind the near pupil response. Whereas Backer and Ogle ${ }^{32}$ argued that fusional vergence was the causative factor. Kasthurirangan and Glasser ${ }^{33}$ support the theory that the near pupil response is elicited by an increase in accommodative stimulus but did not exclude external influencing factors proposed by the above mentioned authors. It should be noted that under low accommodative stimuli $(\leq 1 \mathrm{D})$ pupillary reaction was insignificant or absent in young patients (aged 23-26 years) ${ }^{30,33}$. This finding would suggest that an increase in depth of focus for young patients with a low accommodative stimulus and response $(\leq 1 \mathrm{D})$ does not occur. For larger accommodative stimuli $(\geq 2 \mathrm{D})$ the amount of pupil constriction increases linearly with the increase in accommodative response to the stimuli, the mean value being $0.58 \mathrm{mmD}^{-1}$, until maximum pupil constriction is reached ${ }^{33}$. The pupil and ciliary body both receive input from the parasympathetic nervous system causing the pupil to become more miotic as the ciliary body contracts and accommodative response increases ${ }^{33}$. Horner's Syndrome is characterized by anisocoria due to the miosis of the affected eye which does not dilate at the same rate as the normal unaffected eye but does exhibit a normal near pupil response and a normal response to light stimuli. The adverse effects are due to a lesion to the sympathetic innervation to the affected eye. A possible near point symptom experienced by these patients is an overactive accommodative response to proximal stimuli ${ }^{25}$.

\section{Afferent pupillary defects}

An afferent pupillary defect occurs as a result of a fully lesioned second cranial nerve which results in an amaurotic pupil ${ }^{25}$. The affected eye is completely blind to all light stimuli. However, the two eyes still present with equal pupil diameters when both eyes are simultaneously subjected to the same light stimuli, and during the near pupil response reflex ${ }^{25}$. Homonymous hemianopic visual field defects can give rise to an afferent pupillary defect only if it is approaching or at completion ${ }^{34}$. A relative afferent pupillary defect (the so-called 
Marcus Gunn pupil ${ }^{25}$ ) is caused by an incomplete optic nerve lesion ${ }^{21}$. Although there are other possible causative factors which can lead to relative afferent pupillary defects. Lam and Thompson ${ }^{35}$ discovered that even dense ocular media do not entirely occlude the retina from light stimulus. The light is just extensively scattered. They noted that a slight relative afferent pupilary defect might be detected in certain cases but the near pupil response is unaffected. Abrams and Knighton ${ }^{36}$ concluded that dense anterior chamber and vitreous hemorrhages will give rise to a full relative afferent pupillary defect. Thompson and Corbett ${ }^{37}$ as well as Newsome et $a l^{29}$, discovered that severe retinal hemorrhaging, as well as photoreceptor, bipolar cell, Mueller cell and retinal pigment epithelium damage resulting in very poor visual acuity (worse than 0.1 in decimal notation) also gives rise to a full relative afferent pupillary defect.

Adies tonic pupil is a syndrome that occurs when the postganglionic innervation to the constrictor pupillae and ciliary muscle is affected. This problem is characterized by light-near dissociation which implies that the ipsilateral pupil or pupils does not respond to light stimuli but responds to near or proximal fixation objects. Although this near pupil response is usually sluggish, it is evident that the response is intact ${ }^{38}$.

Light induced pupil responses are elicited evenly by both pupils. This is due to the fact that before the efferent pupillary reflex loop is reached the fibres hemidecussate. Therefore, if an afferent pupillary reflex loop lesion is present, it will not result in anisocoria ${ }^{21}$. The near pupillary response (pupil constriction) observed when attempting to view a near object is also equal in both eyes due to the pupillary reflex arc receiving information from both cerebral hemispheres ${ }^{29}$. Hence, only an efferent loop lesion will cause the patient to report a marked difference in pupil size between the two eyes ${ }^{2,21}$. The use of the direct, consensual and swinging flash light tests can be used to differentiate between the afferent papillary loop stimulation of either one or both eyes ${ }^{21}$.

\section{Depth offocus}

Accommodation is driven by blur but it is also influenced by depth of focus ${ }^{39}$. The perception of blur can be seen as an unfocused image on the retina which triggers accommodation. Depth of focus can be thought of as the amount of blur that will be accepted by the eye. As retinal eccentricity increases so does the depth of focus which suggests an increase in blur tolerance ${ }^{39}$. When viewing a distant object the retinal image will fall within the distal boundary of the depth of focus while the retinal image will fall within the anterior boundary of the depth of focus while viewing a near target ${ }^{40}$. As the image falls within these boundaries the accommodative response will react in the form of a lag or a lead ${ }^{40}$. At the dark focus the accommodative demand and response are equal $^{40}$.

Depth of focus decreases with increased luminance, increased target detail and increased pupil size. An increased depth of focus is due to low contrast, shorter wavelengths, retinal eccentricity, increased accommodative error and increased age, while a smaller depth of focus is found with a better visual acuity which suggests more accurate accommodation. More accurate accommodation can also be achieved by enhancing the depth of focus with visual therapy ${ }^{40}$. Depth of focus varies with direction of gaze and thus the accommodative response varies as well ${ }^{41}$. The accommodative response to blur also serves as a way to judge the distance of objects ${ }^{41}$. A patient that exhibits a greater degree of near pupillary response will in turn experience a greater depth of focus at the retinal plain ${ }^{42}$. This then allows for a less accurate accommodative response because the image quality is perceived to be the same throughout the range of the depth of focus ${ }^{39}$. Hence, early presbyopic patients that present with a highly miotic pupil under near conditions will experience less blur symptoms than if that same patient's pupil was more dilated. Increased depth of focus is, however, secondary to the miotic shift of pupillary miosis during aging ${ }^{22}$. This is because the primary function of the pupil is to control retinal illuminance ${ }^{43}$. Older patients also tend to become less sensitive to blur, causing non-corresponding objective and subjective measures of depth of focus ${ }^{44}$. In addition, the dioptric value of depth of focus increases in the retinal periphery ${ }^{39,45}$. This allows eccentric viewers to require less accommodative response for near stimuli $^{39}$. 


\section{Conclusion}

Problems relating to the near triad can present in a variety of ways. These problems are not necessarily independent of each other but can be interrelated. For example, convergence insufficiency increasing the accommodative response. In order for the point focus to fall on the retina the accommodative system must accurately respond to the visual stimulus presented. Fusional alignment is important to maintain single binocular vision and pupil constriction aids the accommodative process by increasing the depth of focus. Accommodative and convergence dysfunctions can be treated with visual corrections and/or therapies. There is substantial evidence to confirm the synkinesis between the three systems although the neurological link between them is as yet not readily understood.

\section{References}

1. Von Noorden GK, Campos EC. Binocular Vision and Ocular Motility. $6^{\text {th }}$ ed. London: Mosby, 2002.

2. $\quad$ Benjamin WJ. Borish's Clinical Refraction. $1^{\text {st }}$ Ed. Philadelphia: WB Saunders Co, 1998.

3. Silverstone L, Rosenthal F. The Lighthouse Handbook on Vision Impairment and Vision Rehabilitation. Vol 1: Vision Impairment. Oxford: University Press, 2000.

4. Dubbelman M, Van der Heijde GL, Weeber HA. Change in shape of the aging human crystalline lens with accommodation. Vision Research 200545 117-132.

5. Cach P, Garcia A, Lara F, Segui M. Diagnostic signs of accommodative insufficiency. Optometry and Vision Science 200279 614-620.

6. Scheimann MM, Rouse MW. Optometric Management of Learning-related Vision Problems. $2^{\text {nd }}$ ed. Missouri: Mosby, 2006.

7. Rutstein RP, Marsh-Tootle W. Acquired unilateral visual loss attributed to an accommodative spasm. Optometry and Vision Science 200178 492-495.

8. Prakash G, Sharma N, Sharma P, Choudhary V, Titiyal J. Accommodative spasm after laser-assisted in situ keratomileusis. American Journal of Ophthalmology 2004134540.

9. Ninomiya S, Fujikado T, Kuroda T, Maeda N, Tano Y, Hirohara Y, Mihashi T. Wavefront analysis in eyes with accommodative spasm. American Journal of Ophthalmology 2003136 1161-1163.

10. Buehren T, Collins MJ. Accommodation stimulus-response function and retinal image quality. Vision Research $2006461633-1645$.

11. Currie DC, Manny RE. The development of accommodation. Vision Research 199737 1525-1533.

12. Al-Qurainy IA. Convergence insufficiency and failure of accommodation following midfacial trauma. British Journal of Oral and Maxillofacial Surgery 199532 71-75.

13. Firth AY. Adie syndrome: evidence for refractive error and accommodative asymmetry as the cause of amblyopia. American Journal of Ophthalmology 1999 12 118-119.

14. He CJ, Gwiazda J, Thorn F, Held R, Vera-Diaz FA. The association of wavefront aberration and accommodative lag in myopes. Vision Research 200545 285-290.

15. Tsukamoto M, Takayuki N, Nishino J, Hara Y, Uozato H, Saishin M. Accommodation causes with-the-rule astigmatism in emmetropes. Optometry and Vision Science 200077 150-155.

16. Blehm C, Vishnu S, Khattak A, Mitra S, Yee RW. Computer vision syndrome: a review. Survey of Ophthalmology $200550253-262$.

17. Marran L, Schor CM. Lens induced aniso-accommodation. Vision Research 199838 3601-3619.

18. Evans B, Doshi S. Binocular Vision and Orthoptics. Oxford: Butterworth-Heinemann, 2001.

19. David Pickwell. Binocular Vision In: Buckingham T (Ed). Visual Problems in Childhood. Oxford: ButterworthHeinemann, 1993.

20. $\quad$ Griffin JR. Binocular Anomalies Procedures for Vision Therapy. $2^{\text {nd }}$ ed. Chicago: Professional press Inc, 1982.

21. Trobe JD. The Neurology of Vision. Oxford: University Press, 2001.

22. Kasthurirangan S, Glasser A. Age related changes in the characteristics of the near pupil response. Vision Research 200646 1393-1403.

23. Gwiazda J, Thorn F, Held R. Accommodation, accommodative convergence, and response AC/A ratios before and at the onset of myopia in children. Optometry and Vision Science 200582 273-278. 
24. Goss DA. Ocular Accommodation, Convergence, and Fixation Disparity. New York: Professional Press Books Fairchild Publications, 1986.

25. Kanski JJ. Clinical Ophthalmology: a Systemic Approach. $5^{\text {th }}$ ed. London: Butterworth-Heinemann, 2003.

26. Scheiman M, Cooper J, Mitchell L, de Land P, Cotter S, Borsting E, London R, Rouse M. A survey of treatment modalities for convergence insufficiency. Optometry and Vision Science 200279 151-157.

27. Borsting EJ, Rouse MW, Mitchell GL, Scheiman M, Cotter SA, Cooper J, Kulp MT, London R. Validity and reliability of the revised convergence insufficiency symptom survey in children aged 9 to 18 years. Optometry and Vision Science 200380 832-838.

28. Kardon RH. Anatomy and Physiology of the Pupil. In: Miller N.R. Newman N.J, eds. Walsh and Hoyt's Clinical Neuro-Ophthalmology. $5^{\text {th }}$ ed Vol 1 Baltimore: Williams and Wilkins 847-897, 1998.

29. Weinstein JM. Anatomy and Physiology of accommodation. In Miller N.R. Newman N.J, eds. Walsh and Hoyt's Clinical Neuro-Ophthalmology $5^{\text {th }}$ Ed Vol1 Baltimore: Williams and Wilkins 899-915, 1998.

30. Marg E, Morgan MW Jr. Further investigation of the pupillary near reflex: the effect of accommodation, fusional convergence and the proximity factor on pupillary diameter. American Journal of Optometry and Archives of American Academy of Optometry 195027 217-225.

31. Phillips NJ, Winn B, Gilmartin B. Absence of pupil response to blur-driven accommodation. Vision Research 199232 1775-1779.

32. Backer WD, Ogle KN. Pupillary response to fusional eye movements. American Journal of Ophthalmology 1964 $58743-756$.

33. Kasthuriranga S, Glasser A. Characteristics of pupil responses during far-to-near and near-to-far accommodation. Ophthalmic and Physiological Optics 200525 328-339.

34. Miller NR. Walsh and Hoyt's Clinical Neuro-Ophthalmology. $4^{\text {th }}$ ed. Vol 1. Baltimore: Williams and Wilkins, 1982.

35. Lam BL, Thompson HS. A unilateral cataract produces a relative afferent pupil defect in the contra lateral eye. Ophthalmology 199097 334-338.

36. Abrams GW, Knighton RW. Falsely extinguished bright-flash electroretinogram: its association with dense vitreous hemorrhage. Archives of Ophthalmology 1982100 1427-1429.

37. Thompson HS, Corbett JJ. Asymmetry of pupillomotor input. Eye 1991 5 36-39.

38. Loewy AD. Neural regulation of the pupil. In: Brooks CM, Koizumi K, Sato A (Eds). Integrative Functions of the Autonomic Nervous System. Tokyo: Elsevier/ North-Holland Biomedical Press pp 131-141, 1979.

39. Wang B, Ciuffreda KJ. Depth-of-focus of the human eye in the near retinal periphery. Vision Research 200444 $1115-1125$.

40. Wang B, Ciuffreda KJ. Depth-of-focus of the human eye: theory and clinical implications. Survey of Ophthalmology 200652 75-85.

41. Ciuffreda KJ, Wang B, Vasudevan B. Conceptual model of human blur perception. Vision Research 200747 1245-1252.

42. Campbell FW. The depth of field of the human eye. Optica Acta 19574 157-164.

43. Loewenfeld IE. Thompson's review of The Pupil: Anatomy, Physiology \& Clinical Implication, Vol I and II. American Journal of Ophthalmology 1993116 117-119.

44. Mordi JA, Ciuffenda KJ. Static aspects of accommodation: age and presbyopia. Vision Research 199838 16431653.

45. Ronchi L, Moselini G. Depth of focus in peripheral vision. Ophthalmic Research 19757 152-157. 